\section{Pantothenate kinase-associated neurodegeneration: Clinical aspects, diagnosis and treatments}

\author{
Saeed Razmeh, ${ }^{1}$ Amir Hassan Habibi, ${ }^{2}$ \\ Maryam Orooji, ${ }^{2}$ Elham Alizadeh, ${ }^{2}$ \\ Karim Moradiankokhdan, ${ }^{3}$ \\ Behroz Razmeh ${ }^{3}$ \\ 1 Yasuj University of Medical Sciences, \\ Yasuj; 2Iran University of Medical \\ Sciences, Tehran; ${ }^{3}$ Kermanshah \\ University of Medical Sciences, \\ Kermanshah, Iran
}

\begin{abstract}
Pantothenate Kinase-Associated Neurodegeneration (PKAN) is an autosomal recessive disorder characterized by a mutation in the PANK2 gene. The clinical presentation may range from only speech disorder to severe generalized dystonia, spasticity, Visual loss, dysphagia and dementia. The hallmark of this disease is eyes of the tiger sign in the medial aspect of bilateral globus pallidus on T2-weighted MRI that is a hyperintense lesion surrounded by hypointensity. Common treatments for PKAN disease include anticholinergics, botulinum toxin, Oral and Intrathecal baclofen, Iron chelation drugs and surgical procedures such as ablative pallidotomy or thalamotomy, Deep brain stimulation. There are many controversies about the pathogenesis and treatment of this disease, and in recent years interesting studies have been done on PKAN disease and other similar diseases. This review summarizes the clinical presentation, etiology, imaging modalities and treatment.
\end{abstract}

\section{Introduction}

Neurodegeneration with brain iron accumulation (NBIA) is a wide number of neurodegenerative disorders characterized by iron accumulation in the brain, especially basal ganglia, substantia nigari, hippocampus and cerebellar nuclei.1,2 The typical presentation of NBIA disorder includes dystonia, Parkinsonism, choreoathetosis, mental dysfunction, spasticity, visual disturbance and bulbar dysfunction. ${ }^{3}$ The most common form of NBIA is PKAN also known as NBIA-1 that is an autosomal recessive disorder characterized by a mutation in the PANK 2 at locus 20 P12. ${ }^{3}$ This disease also has various psychiatry symp- toms.4-6 This review aims to describe the clinical manifestation, pathophysiology and the treatment of PKAN disease.

\section{Materials and Methods}

\section{Etiology}

The iron normally accumulated in the basal ganglia, hippocampus, cerebellar nuclei and another subcortical region. ${ }^{7}$ The PKAN characterized by a mutation in the PANK 2 that codes the pantothenate kinase 2. Mutations are missense, duplication, deletion, splice site mutation and deletion of exon. ${ }^{8}$ This protein kinase is responsible for the phosphorylation of pantothenate and this product combine with cysteine. The cysteine increase in the globus pallidus of patients with PKAN that in association with iron produce free radicals and cell damage. 9,10 The Mutations of PANK2 also found in patients with HARP (hypoprebetalipoproteinemia, acanthocytosis, retinitis pigmentosa, and pallidal degeneration). The HARP is rare disease and has mental decline, abnormal movement and vision loss. ${ }^{11}$

\section{Diagnosis}

Today, the PKAN disease is diagnosed by magnetic resonance imaging and confirmed with the genetic test. 12 Susceptibility weighted imaging (SWI) and field dependent R2 increase (FDRI) are the choice methods for the diagnosis of this disease and FDRI is more sensitive than SWI for detection of brain iron. ${ }^{13}$ We can also use the cranial sonography that detects the over load of iron in midbrain. ${ }^{14}$ The hallmark of this disease in the BRAIN MRI is eye of the tiger sign. The eye of the tiger is a hyperintense lesion surrounded by hypointensity in the medial aspect of bilateral globus pallidus on T2weighted MRI. Central hyperintensity shows gliosis in globus pallidus and the surrounding hypointensity showing iron deposition (Figures 1 and 2). ${ }^{15}$ All of the patients with eye-of-the-tiger sign don't have PANK2 mutation, Streckera et al. report a patient with multiple system atrophy (MSA) and systemic lupus erythematodes that has typical Eye of the Tiger sign in T2-weighted MRI. ${ }^{16}$ Chaw-Liang Chang also reported a patient with cervical dystonia that has Eye of the Tiger without evidence of PANK2 gene mutation. ${ }^{17}$ This sign also reported in a patient with mitochondrial membrane protein associatedneurodegeneration (MPAN). ${ }^{18}$ The brain MRI may be normal in the Early Stages of Classic Pantothenate Kinase Associated Neurodegeneration and after the progression of disease, the Eye of the Tiger sign appear. 19
Correspondence: Saeed Razmeh, Yasuj University of Medical Sciences, Kohgiluyeh and Boyer-Ahmad Province, Yasuj, Shahid Motahari Blvd, Iran.

E-mail: srazmeh@yahoo.com

Key words: Pantothenate Kinase-Associated Neurodegeneration, Neurodegeneration with brain iron accumulation.

Contributions: the authors contributed equally.

Conflict of interest: the authors declare no potential conflict of interest.

Funding: none.

Received for publication: 30 November 2017. Revision received: 29 December 2017.

Accepted for publication: 4 February 2018.

This work is licensed under a Creative Commons Attribution NonCommercial 4.0 License (CC BY-NC 4.0).

CC Copyright S. Razmeh et al., 2018

Licensee PAGEPress, Italy

Neurology International 2018; 10:7516

doi:10.4081/ni.2018.7516

\section{Classification and presentations}

It separated into classic and atypical form. The classic form starts in early childhood with rapid progression of symptoms including Parkinsonism, dystonia, cognitive decline and dementia, dysarthria, spasticity, seizure, optic atrophy, pigmentary retinopathy that disable the patient very sever. Atypical form of disease has later onset and less common than classic form and it may not be recognized by aging and has milder symptoms with slow progression in compared to classic type. ${ }^{20,21}$ The neuropsychiatric disorder includes ObsessiveCompulsive Disorder (OCD), depression and schizophrenia-like psychosis. ${ }^{22}$ In study that assessed the Intellectual and adaptive behavior functioning 16 children and adults with PKAN, there is varied cognitive expression, also there was an inverse correlation between the onset of the disease and the cognitive impairment. ${ }^{23}$ The ophthalmologic manifestation is variable and includes saccadic pursuits, convergence impairment, vertical hypermetric saccades, square wave jerks and pigmentary retinopathy. 24

\section{Treatment}

\section{Medications}

There is currently no suitable therapy 


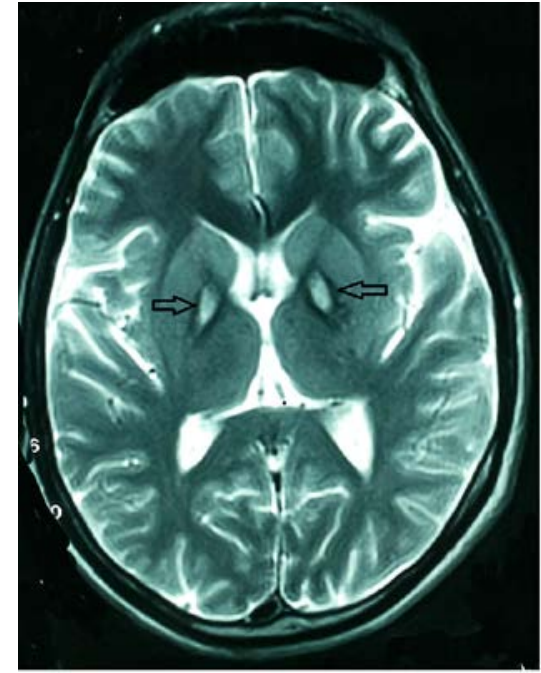

Figure 1. T2 weighted Brain magnetic resonance imaging of the patient that shows hyperintense lesion surrounded by hypointensity in the medial aspect of bilateral globus pallidus (eye-of-the-tiger).

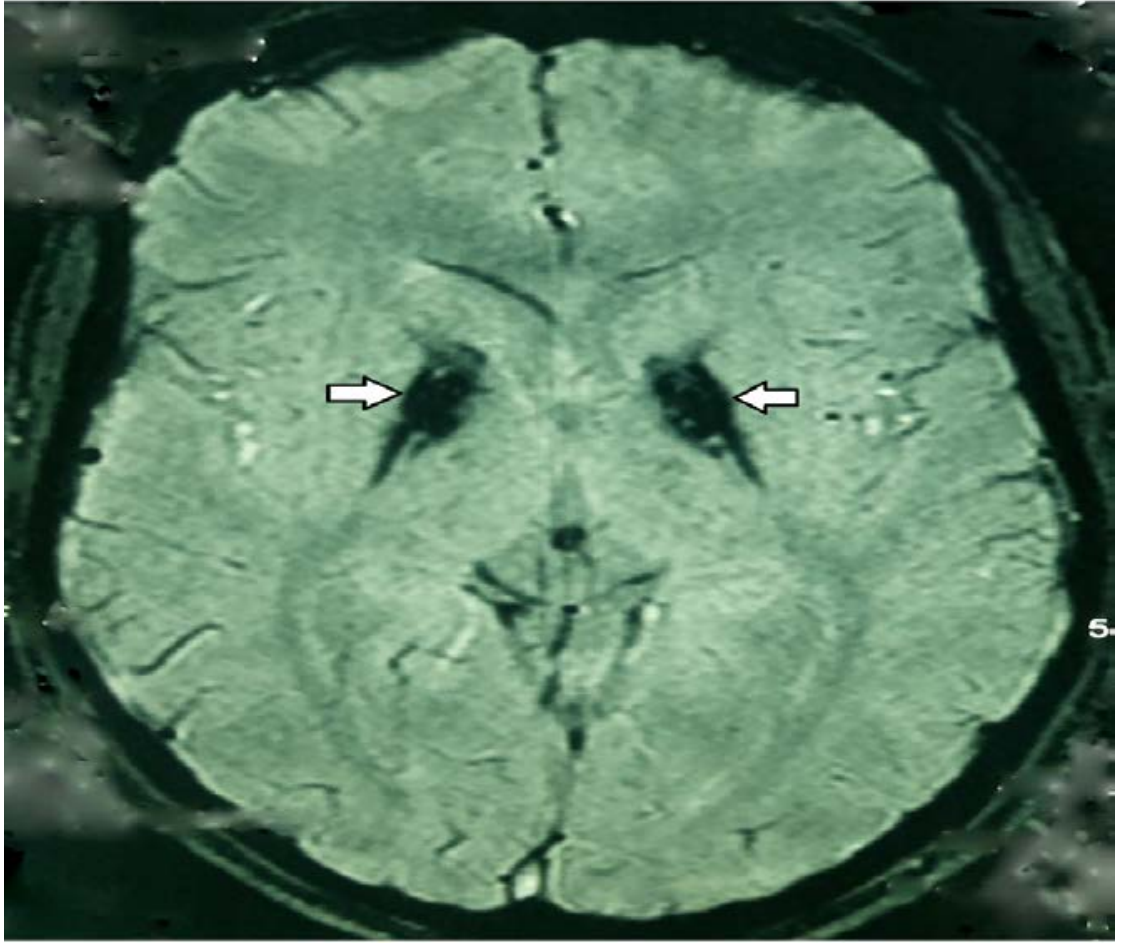

Figure 2. Susceptibility weighted imaging (SWI) of the PKAN patient that shows hypointense lesion in the medial aspect of bilateral globus pallidus. for PKAN patients and different available drugs don't effect on disease progression. ${ }^{25}$ Dystonia can be profoundly disabling in the affected patients and progresses over time and involves many parts of the body. It usually treated with anticholinergics, botulinum toxin, Oral and Intrathecal baclofen, benzodiazepines, Clonidine, gabapentin, pregabalin, tetrabenazine and other antispasticity drugs, alone or in combination. 26,27 Vitamin B5 (pantothenate) may be able to improve PKAN patients by increasing levels of CoA and recovery of mitochondrial dysfunction. In mouse models of PKAN with a high-fat ketogenic diet, this drug was able to prevent some neurological symptoms, But to investigate its effects, clinical trial studies should be conducted in the future. $28-30$

\section{Surgery}

Surgical procedures such as Deep brain stimulation are now used for PKAN disease. The benefit effect of Deep brain stimulation, can occur quickly after surgery, But due to the progressive nature of the disease, symptoms may reappear after a while and the disease progresses again. ${ }^{31,32}$ In addition to Deep brain stimulation, other surgical procedures including Ablative pallidotomy or thalmotomy can also be used, although these surgical procedures are currently less commonly used and replaced by Deep brain stimulation. ${ }^{33}$ In PKAN patients that have severe dystonia, spasticity and pain, intrathecal baclofen (ITB) pump can alleviate the symptoms. ${ }^{34}$ Blair Ford et al. shows intrathecal baclofen is more effective if the dystonia is associated with spasticity and pain 35 it can also use intraventricular. In a study of Albright et al on ten patients with severe generalized secondary and heredodegenerative dystonia, Intraventricular baclofen was effective on dystonia. ${ }^{36}$

\section{Iron chelation}

The drugs currently used include deferiprone, deferoxamine and deferasirox. ${ }^{37}$ Nowadays, Deferiprone have attracted the attention of many researchers. It can cross the blood-brain barrier to remove iron and prevent its accumulation. ${ }^{38,39}$ In studies that investigated the effect of deferiprone on the NBIA patients, the use of this drug was safe and without adverse effects. In these studies, deferiprone reduced iron load in brain imaging and in clinical evaluations, variable results were obtained which requires further investigation in the future. 40,41

\section{References}

1. Hayflick SJ. Neurodegeneration with brain iron accumulation: from genes to pathogenesis. Semin Pediatr Neurol 2006; 13:182-5.

2. Thomas M, Hayflick SJ, Jankovic J. Clinical heterogeneity of neurodegeneration with brain iron accumulation and pantothenatekinase-associated neurodegeneration. Movement Disord 2004; 19:36-42.

3. Hogarth P. Neurodegeneration with brain iron accumulation: diagnosis and management. J Mov Disord 2015;8:113.

4. Hayflick SJ, Westaway SK, Levinson $\mathrm{B}$, et al. Genetic, clinical, and radiographic delineation of PKAN disease. N Engl J Med 2003;348:33-40.

5. Gregory A, Polster BJ, Hayflick SJ. Clinical and genetic delineation of neurodegeneration with brain iron accumulation. J Med Genet 2009;46:73-80.

6. Pawar Y, Kalra G. A case of PKAN disease presenting as catatonic schizophrenia. Indian J Psychiatry 2013;55:386-9.

7. Hayflick SJ, Westaway SK, Levinsonetal B. Genetic clinical, and radiographic delineation of PKAN disease. New Engl J Med 2003;348:33-40.

8. Levi S, Finazzi D. Neurodegeneration with brain iron accumulation: update on pathogenic mechanisms. Front Pharmacol 2014;5:99.

9. Hayflick SJ, Westaway SK, Levinson B, 
et al. Genetic, clinical, and radiographic delineation of PKAN disease. N Engl J Med 2003;348:33-40.

10. Gregory A, Westaway SK, Holm IE, et al. Neurodegeneration associated with genetic defects in phospholipase $\mathrm{A}(2)$. Neurology 2008;71:1402-9.

11. Ching KH, Westaway SK, Gitschier J, et al. HARP syndrome is allelic with pantothenate kinase-associated neurodegeneration. Neurology 2002;58: 1673-4.

12. Kruer MC, Boddaert N, Schneider SA, et al. Neuroimaging features of neurodegeneration with brain iron accumulation. Am J Neuroradiol 2012;33:40714.

13. Schipper HM. Neurodegeneration with brain iron accumulation - Clinical syndromes and neuroimaging. Biochim Biophys Acta 2012;1822:350-60.

14. Liman J, Wellmer A, Rostasy K, et al. Transcranial ultrasound in neurodegeneration with brain iron accumulation (NBIA). Eur J Paediatr Neurol 2012;16: 175-8.

15. Savoiardo M, Halliday WC, Nardocci $\mathrm{N}$, et al. Hallervorden-Spatz disease: MR and pathologic findings. Am J Neuroradiol 1993;14:155-62.

16. Strecker K, Hesse S, Wegner F, et al. Schneider Eye of the tiger sign in multiple system atrophy. Eur J Neurol 2007;14:e1-2.

17. Chang CL, Lin CM. Eye-of-the-Tiger sign is not Pathognomonic of Pantothenate Kinase-Associated Neurodegeneration in Adult Cases. Brain Behav 2011;1:55-6.

18. Yoganathan S, Sudhakar SV, T. Maya, et al. Eye of tiger sign mimic in an adolescent boy with mitochondrial membrane protein associated neurodegeneration (MPAN). Brain Dev 2016;38:5169.

19. Chiapparini L, Savoiardo M, D’Arrigo $\mathrm{S}$, et al. The eye-of-the-tiger sign may be absent in the early stages of classic pantothenate kinase associated neurodegeneration. Neuropediatrics 2011;42:159-62.

20. Amaral L, Gaddikeri S, Chapman PR, et al. Neurodegeneration with brain iron accumulation: clinicoradiological approach to diagnosis. J Neuroimaging
2015;25:539-51.

21. Meyer E, Kurian MA, Hayflick SJ. Neurodegeneration with brain iron accumulation: enetic diversity and pathophysiological mechanisms. Ann Rev Genomics Hum Genet 2015;16:257-79.

22. Loring DW, Sethi KD, Lee GP, Meador KJ. Neuropsychological performance in PKAN disease : a report of two cases. Neuropsychology 1990;4:191-9.

23. Freeman K, Gregory A, Turner A, et al. Intellectual and adaptive behaviour functioning in pantothenate kinaseassociated neurodegeneration. $\mathrm{J}$ Intellect Disabil Res 2007;51:417-26.

24. Egan RA, Weleber RG, Hogarth P, et al. Neuroopthamologic and electroreinographic finding in pantothenate kinase associated neurodegeneration. Am J Ophtalmol 2005;140:167-274.

25. Schneider SA, Bhatia KP, Hardy J. Complicated recessive dystonia Parkinsonism syndromes. Mov Disord 2009;24:490-9.

26. Zorzi G, Zibordi F, Chiapparini L, Nardocci N. Therapeutic Advances in Neurodegeneration With Brain Iron Accumulation. Semin Pediatr Neurol 2012;19:82-6.

27. Cloud LJ, Jinnah H. Treatment strategies for dystonia. Exp Opin Pharmacother 2010;11:5-15.

28. Kuo YM, Hayflick SJ, Gitschier J. Deprivation of pantothenic acid elicits a movement disorder and azoospermia in a mouse model of pantothenate kinaseassociated neurodegeneration. J Inherit Metab Dis 2007;30:310.

29. Brunetti D, Dusi S, Morbin M, et al. Pantothenate kinase-associated neurodegeneration: altered mitochondria membrane potential and defective respiration in Pank2 knock-out mouse model. Hum Mol Genet 2012;21:5294305.

30. Brunetti D, Dusi S, Giordano C, et al, Pantethine treatment is effective in recovering the disease phenotype induced by ketogenic diet in a pantothenate kinase-associated neurodegeneration mouse model. Brain 2014;137:57-68.

31. Umemura A, Jaggi JL, Dolinskas CA, et al. Pallidal deep brain stimulation for longstanding severe generalized dystonia in PKAN disease: case report. J Neurosurg 2004;100:706-9.

32. Balas I, Kovacs N, Hollody K. Staged bilateral stereotactic pallidothalamotomy for life-threatening dystonia in a child with PKAN disease, Mov Disord 2006;21:82-5.

33. Justesen CR, Penn RD, Kroin JS, Egel RT. Stereotactic pallidotomy in a child with PKAN disease. Case report, J. Neurosurg 1999;90:551-4.

34. Albright AL, Barry M, Fasick P, et al. Continuous intrathecal baclofen infusion for symptomatic generalized dystonia [clinical studies]. Neurosurgery 1996;38:934-9.

35. Ford B, Greene P, Louis ED, et al. Use of intrathecal baclofen in the treatment of patients with dystonia. Arch Neurol 1996;53:1241-6.

36. Albright AL, Ferson SS. Intraventricular baclofen for dystonia: techniques and outcomes. Clinical article. J Neurosurg Pediatr 2009;3:11-4.

37. Gomber S, Saxena R, Madan N. Comparative efficacy of desferrioxamine, deferiprone and in combination on iron chelation in thalassemic children. Indian Pediatr 2004;41: 21-7.

38. Boddaert N, Le Quan Sang KH, Rötig A, et al. Selective iron chelation in Friedreich ataxia: biologic and clinical implications. Blood 2007;110: 401-8.

39. Olivieri NF, Brittenham GM, McLaren $\mathrm{CE}$, et al. Long-term safety and effectiveness of iron-chelation therapy with deferiprone for thalassemia major. New Engl J Med 1998;339:417-23

40. Zorzi G, Zibordi F, Chiapparini L, et al. Iron-related MRI images in patients with Pantothenate Kinase-Associated Neurodegeneration (PKAN) treated with Deferiprone: results of a phase II pilot trial. Mov Disord 2011;26:1756-9.

41. Cossu G, Abruzzese G, Matta G, et al. Efficacy and safety of deferiprone for the treatment of pantothenate kinaseassociated neurodegeneration (PKAN) and neurodegeneration with brain iron accumulation (NBIA): results from a four years follow-up. Parkinsonism Relat Disord 2014;20:651-4. 